\title{
FUE Donor Site Ischemia and Necrosis
}

\author{
Cagatay Sezgin, MD Adana, Turkey cagataysezgin66@gmail.com
}

The follicular unit extraction (FUE) method for hair transplantation is becoming more popular because of the patient preferences and it has the advantages of rapid recovery, comfortable post-operative period, and, for some patients, increasing donor supply. However, FUE donor site complications are rare in the literature. ${ }^{1}$

I want to present and discuss one of these rare donor site complications that I have encountered: a patient with donor site ischemia and necrosis, which progressed, increased during the extraction period, and than healed with a scarring alopecia area.

\section{Case Study}

The 39-year-old male patient was a Norwood type V. The patient reported no known disease and no medications and/or supplements. He had been smoking heavily (20-40 cigarettes/ day) since his twenties. His preoperative hemogram test had shown HB: $17 \mathrm{~g} / \mathrm{dL}, \mathrm{HCT}: 51.5 \%$ (upper limit of normal 52).

We planned two FUE sessions. In the first session, 2,720 grafts were harvested and transplanted. We used lidocaine $2 \%$ with 1:100000 epinephrine for donor site anaesthesia. A total of 6-8cc was administered to the entire donor area. We began harvesting from the right temporal region moving around the head and finishing in the left temporal region.

It took approximately 3 hours and 15 minutes to harvest the grafts with a micromotor using $0.9 \mathrm{~mm}$ sharp punch inserted to an average depth of $2-3 \mathrm{~mm}$. After finishing the extraction, I noticed an ischemic area on the right temporal region. We did the routine dressing of the donor area, and after 30 minutes began to create recipient sites followed by graft placement. After completing placement, I examined the ischemic region again and saw some resolution, but also noted that some points had worsened (Figure 1).

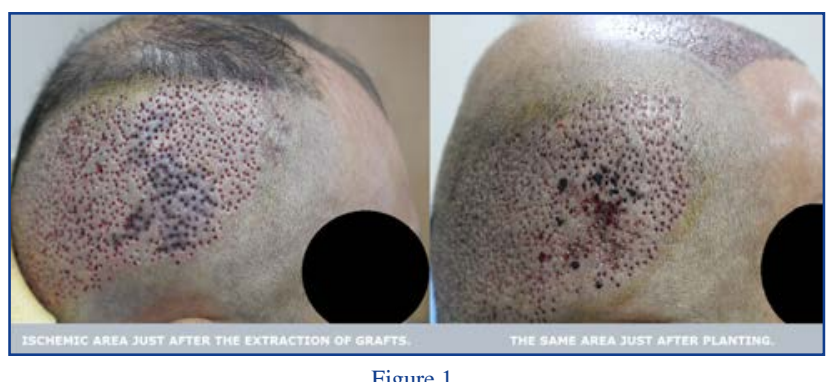

I prescribed pentoxifylline $600 \mathrm{mg}$ twice per day for 7 days post-operatively, and warned the patient not to lean this side of his head on the pillow while sleeping. After two months, part of the ischemic area reduced and healed, but the necrotic area healed with a $1.5-2 \mathrm{~cm}$ wide scar (Figure 2).

\section{Discussion}

The possible factors that might have lead to and/or triggered the isch-

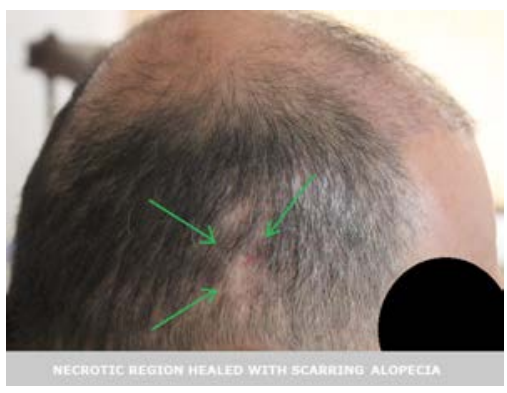

Figure 2. emia and necrosis in this case are heavy smoking, very thin subcutaneous tissue, and prolonged compression (lying on this side of the head for a long period during graft extraction). Although his HCT was only high normal, it must be noted that high HCTs in heavy smokers are associated with increased blood viscosity, ${ }^{2}$ another potential risk factor for ischemia.

\section{Conclusion}

Heavy smoking, thin subcutaneous tissue, prolonged pressure, and perhaps increased blood viscosity together and/or solely may reduce tissue blood circulation that may lead to tissue ischemia and necrosis in FUE harvesting.

\section{References}

1. Karaçal, N., et al. Necrosis of the donor site after hair restoration with follicular unit extraction (FUE): a case report. J Plast Reconstr Aesthet Surg. 2012(Apr); 65(4):e87-89.

2. Alkan, F.A., et al. The evaluation of plasma viscosity and endothelial dysfunction in smoking individuals. Clin Hemorheol Microcirc. 2013 Oct 29. [Epub ahead of print]

Editor's Note: I asked Dr. Sezgin if there had been any arterial bleeding at the time of anesthesia injection or punch insertion in the area of necrosis. He reported there was none. - RT

\section{Commentary:}

James A. Harris, MD, FACS Greenwood Village, Colorado USAJHairSMD@aol.com

This is likely the first reported case of donor area necrosis after an FUE procedure in the literature. Dr. Sezgin has reviewed the pertinent risk factors and it is apparent that it should be routine to ask patients about these factors. The question of a high hematocrit may be a little difficult to answer as pre-operative laboratory testing is not routine in all offices. Smoking has been identified as a risk factor in recipient area necrosis.

It seems that some factors that might place the patient at obvious risk for donor necrosis did not play a role in this case. I'm referring to excessive amounts of high concentration epinephrine as a tumescent solution to aid in graft dissection, deep insertion of sharp punches, high density extractions, and the use of large punches. The author does not describe the use of tumescence in this case so this would not have been a contributing factor.

The total linear trauma from the dissections would be related to the size of the punch and number of attempts. The author states that the procedure involved 2,720 extraction attempts, which does not seem excessive for the apparent size of the donor area. In addition, a .09mm punch was used, which is not "large" for FUE cases. Just as the recipient area is subject to excessive trauma and at risk for necrosis, one might expect the donor area to have the same risk factors.

Every month we see examples on the internet of surgeons, or their staff, attempting FUE cases of 5,000 to 6,000 grafts. The photos of these cases certainly appear extreme and one can only imagine the complications we don't hear about. I would 


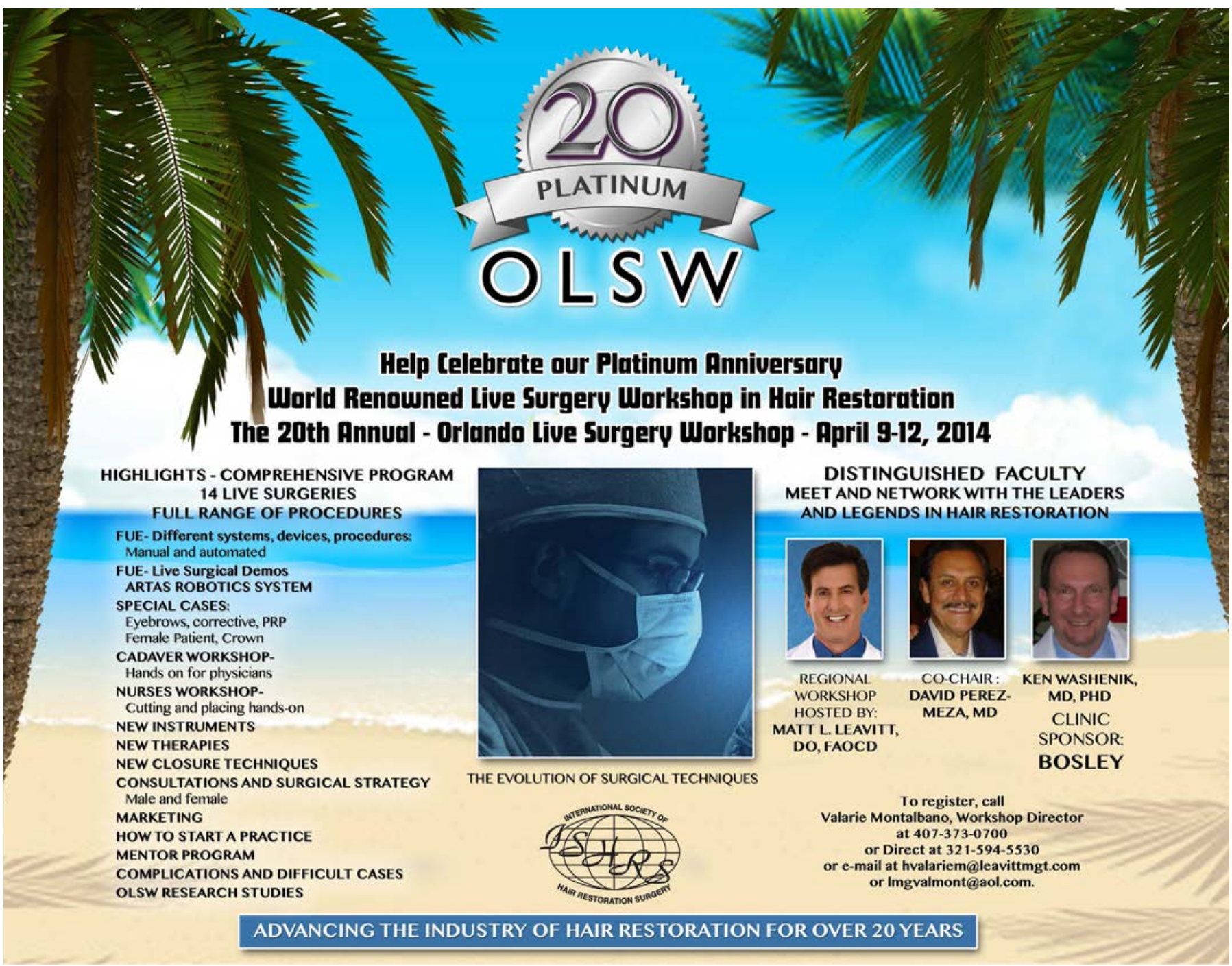

urge physicians performing FUE to be cautious, ask about the risk factors that Dr. Sezgin discusses, and be observant for any indications of blood supply disruption.

In spite of this report, and having performed FUE for 11 years, this is the first time I have heard of a case of donor area necrosis related to FUE. Notwithstanding extraction site hypopigmentation and over harvesting, we are fortu- nate that donor area complications from FUE are relatively rare. As in most areas of hair restoration, we have no data for FUE procedures to indicate what the limits are in terms of total linear trauma, the volume of tissue removed, extraction densities, and punch size limits. It would seem that this area would be a fruitful area of study for the ISHRS FUE Research Committee to consider. 